\title{
The Nature of Cohesion.*
}

I IKE all other branches of physics, the subject of 1 cohesion has been profoundly modified by the advent of wave mechanics. When atoms wore regarded as miniature solar systems, there was no mathematical machinery capable of dealing with their mutual interaction. But now the outlook is different. There is no attempt in wave mechanics to follow the life-history of electrons in atoms. Rather does it give a statistical average of their distribution in space, from which may be inferred the probability that an electron may be found here or found there. Mathematically, if not physically, electrons in atoms are smoared out. Where once was thought a solar system played, is now a shimmering haze.

Wave mechanics does not give the density of these charge distributions directly. It gives instead, when only one electron is being considered, a quantity $\psi$, callod a wave function, which may bo real or complex, and the density $\rho$ of the electron distribution is equal to the square of the modulus of $\psi$ or to $\psi \psi$. The quantity $\psi$ is a function of the co-ordinates of the electron, and these are four in number, thrce being necessary to specify its position in space, and the fourth its direction of spin. The probability of finding the electron in an element of volume $d \tau$ is $\rho d \tau$.

When there aro several electrons in an atom, its configuration may in an approximate theory be specified by a number of wave functions, $\psi_{\alpha}, \psi_{\beta}, \ldots \psi_{\nu}$, each a function of the four co-ordinates of an eloctron. For a reason not yet understood, Nature places a restriction on the choice of these wave functions : no two of them may be the same. This exclusion principle, first enunciated by Pauli, seoms to be of very general validity in physics, and, in particular, accounts in a very remarkable way for many of the observed properties of atoms and molecules.

lf ono of the electrons, say electron 1 , were alone, the probability of finding it in an element of volume $d \tau_{1}$, would be $\Psi_{a}\left(x_{1}\right) \psi_{a}\left(x_{1}\right) d \tau_{1}$ or $\rho_{a}\left(x_{1}\right) d \tau_{1}$, where $x_{1}$ denotes its four co-ordinates. Similarly the probability of finding electron 2 in an element of volume $d \tau_{2}$, if it were alone, would be $\rho_{\beta}\left(x_{2}\right) d \tau_{2}$. It might be supposed that the probability of finding an electron in $d \tau_{1}$, another in $d \tau_{2}$, and so on, simultaneously, were the product of these separate probabilities, namely, $\rho_{a}\left(x_{1}\right)_{\rho_{\beta}}\left(x_{2}\right) \ldots d \tau_{1} d r_{2} \ldots$. But this result is not true. It is untrue because an important principle has been ignored, namely, that all electrons are identical and that interchanges of the electrons may occur without observable effects. Electron 1 may be associated with wave function $\psi_{\beta}$, or in fact with any of the set of wave functions $\psi_{a}$ to $\psi_{\nu}$. The correct expression for the probability may, in certain cases, be expressed quite simply, as has recently been shown by Dirac. It is $\rho d \tau_{1} d \tau_{2} \ldots$. , where $\rho$ is a determinant of $N$ rows and columns, a typical term of which is $\rho\left(x_{j}, x_{k}\right)=\Sigma_{\alpha} \Psi_{\alpha}\left(x_{j}\right) \psi_{\alpha}\left(x_{k}\right)$. This determinantal form for $\rho$ contains the expression given above as its leading term, but there are many other terms not previously given by an atomic theory.

The probability of finding an electron in $d \tau_{1}$ independently of the position of all other electrons is then obtained by integrating $\rho$ over the co-ordinates of all the electrons except one, and may be shown to be $\Sigma_{\alpha \rho_{a}}\left(x_{1}\right) d \tau_{1}$. This expression is the sum of the densities of the probability patterns of the separate electrons, and, moreover, contains the co-ordinates of

* From two lectures delivered recently by Prof. J. E. Lennard-Jones, one on "Cohesion", before the Physical Society of London, and the other on the "Quantum Mechanics of Atoms and Molecules", before the London Mathematical Society. one electron only, so that, apart from the spin coordinate it may be represented in ordinary three dimensional space. It justifies us in constructing a picture of an atom by superimposing the patterns of the individual electrons of which it is composed. Such pictures are very instructive and have been used in a variety of ways with striking success. One important result is that the distribution of electron density in the inert gases and, indeed, in many other atoms is spherically symmetrical, and so the early pictures of them as minute spheres are to some extent justified. The halogen atoms, on the other hand, are not spherical. They are like the familiar meteorological forecast, "Unsettled, with bright intervals".

The importance of the new form for the probability function $\rho$ becomes evident when calculations are made of the energy of electronic systems. Electrons exert electrostatic forces on each other and the average value of the electrostatic energy can only be calculated when we know the probability that any pair of electrons will be at a specified distance apart. $\mathrm{A}$ detailed calculation of the energy of an atom or the interaction energy of two atoms depends then very closely on the probability function $\rho$.

The probability of finding two electrons in specified places independently of the other electrons proves to be $\left\{\rho\left(x_{1}, x_{1}\right) \rho\left(x_{2}, x_{2}\right)-\rho\left(x_{1}, x_{2}\right) \rho\left(x_{2}, x_{1}\right)\right\} d \tau_{1} d \tau_{2}$, with the definitions of $\rho\left(x_{1}, x_{2}\right)$, etc., already given. The mutual energy of two electrons consists then of two terms; the first is the average of $\left(e^{2} / r\right) \rho\left(x_{1}, x_{1}\right) \rho\left(x_{2}, x_{2}\right)$ integrated over the whole space of electrons 1 and 2 ; the second is the average of $\left(e^{2} / r\right) \rho\left(x_{1}, x_{2}\right) \rho\left(x_{2}, x_{1}\right)$. Apart from certain terms of these expressions which cancel, the first represents the Coulomb interaction of the individual distributions of electric chargo $\rho_{\alpha}, \rho_{\beta}$, ete. This may be called the Coulomb energy.

The second term in the above expression is new. It has proved of the greatest importance in the theory of atomic and molecular structure. It is difficult to describe its physical naturo. All that can be said is that it is the natural outcome of introducing two physical concepts into the mathematical scheme, namely, tho principle of the identity of electrons, and the exclusion principle of Pauli. It is sometimes described as the 'exchange' term and the term in the energy expression arising from it as the 'exchange" energy.

The 'exchange' energy depends on the spin of the electrons, while the Coulomb energy does not. It is this property of the exchange term which has made it of so much importance in the theory of atoms and molecules. In a two-electron system, as in an excited helium atom, for example, the electrons may have the same or opposite spins, and, owing to the exchange term, the energy of the states with the same spins is lower in every case than the corresponding states with opposite spin. The hydrogen molecule is another two-electron system, and hore the energy is lowest when the electrons have opposite spin. This appears to be the case in the interaction of most atoms. For certain interatomic distances the energy is lowest, and, therefore, the cohesion greatest, when the electrons of one atorn are 'paired' with those of the other atom. The energy of two interacting nitrogen atoms is highest when the spins of the three outer electrons of the atoms are all of them the same, and lowest when the three electrons of one atom are pairod with those of the other. This latter condition corresponds to the normal nitrogen molecule, held together-as the chemist describes it-with a triple bond.

The pairing of electrons is thus brought into close 
connexion with the valency rules of the chemist, and chemical homopolar forces are elucidated to this extent - that they are seen to be a consequence of the same mathematical and physical principles which have been formulated for other branches of physics. This result may conceivably come to be regarded as one of the greatest achievements of the present formulation of quantum mechanics.

The new theory has been successful in explaining other forces, the nature of which was formerly little understood. For a long time it has been known that atoms, which are chemically inert, exert attractions on each other; so much so, that they aggregate together as liquids and solids if the temperature is lowered sufficiently. These attractive forces, usually called van der Waals forces, have now in one or two simple cases been calculated deductively from the electronic structure of the atoms. These attractive forces seem to be due not to a static polarisation of the atoms by each other but rather to a rapidly fluctuating mutual polarisation. Atoms are not actually continuous distributions of space charge. They are only to be regarded so on the average. When two atoms are subject to each other's influence, the motion of the electrons in one modifies that of the electrons in the other. They tend on the average to move in phase.

Suppose, for the sake of illustration, that an atom were represented by a linear oscillator, that is by an electron vibrating along a line through the nucleus. Such a system requires only one co-ordinate $z$ to specify it. Then the probability-distribution of the electron in its lowest energy state is a Gaussian error curve $\left(e^{-a z^{2}}\right)$, symmetrical about the origin. If two such electronic systems, vibrating along the line joining the nuclei and specified by co-ordinates $z_{1}$ and $z_{2}$, are allowed to interact, the new probability-distribution is not simply a combination of the two un- disturbed probability-distributions of the isolated systems. Calculation shows that as a result of interaction the probability of finding $z_{1}$ and $z_{2}$ with the same sign has increased, while that of finding them with the opposite sign has decreased. Now dipoles which point in the same direction attract, and those which point in the opposite direction repel. The net result is that the two electronic systems on the average attract. The attractive force for this model and for actual atoms proves to be proportional to the inverse seventh power of the distance.

All atoms and molecules exert on each other an attractive field of this type, but usually this attraction is masked by other larger attractive fields. Only in the case of inert gases or saturated molecules does it become predominatingly important. It is likely that many substances, such as the halogen hydrides, are held together in the solid state by forces of this type. There is a growing recognition, too, of the importance of van der Waals fields in many phenomena at surfaces such as in adsorption.

The problem of ionic cohesion largely resolves itself into explaining why some atoms have an affinity for electrons. Such atoms are usually unsymmetrical charge distributions and the nature of the affinity can be understood, though actual calculation of the magnitude is difficult.

Metallic cohesion seems to be due partly to the Coulomb interaction of space charge distributions (the Coulomb attraction referred to above), partly to the ' exchange' phenomenon, and partly to van der Waals attraction. Little is as yet known as to the relative extent to which these various factors contribute to the cohesion of a metal. The general principles seem to be understood. What is now required is a mathematical technique capable of applying them to particular cases.

\section{Selection of Engineering Apprentices.*}

$\mathrm{H}^{\circ}$ W far is it practicable to use psychological tests in the selection of boys for the engineering trade? An attempt to provide an answer to this question has recently been made by the Birmingham Education Committee, under the auspices of which an investigation was conducted by Miss F. P. Allen and Mr. Percival Smith. A report describing the tests used, the methods adopted, and the results obtained has now been published.

Such a report will be of especial value to local education authorities responsible for juvenile employment. Indeed, the Malcolm Committee on Education and Industry has already directed attention to the necessity for this kind of research by authorities upon whom that responsibility rests. The present report deals with one aspect of the wide problem with which such authorities are faced : its authors have also been engaged upon a second experiment, involving still wider issues - the extent to which a series of psychological tests can be of practical use in offering vocational guidance to children when they leave elementary schools.

So far as the engineering trade is concerned, however, one of the first points to be decided was what that all-embracing term meant. In Birmingham, as elsewhere, it covers a multiplicity of occupations, ranging from those followed by practically unskilled manual workers to those needing highly skilled techni-

- City of Birmingham: Education Committee. Selection of skilled Apprentices for the Engineering Trades, Report of Research. By E. Patricia Allen and Percival Smith. Pp. iv-35. (Birmingham: Education Committee, 1931.)

No. 3228 , VoL. 128$]$ cal experts. For the former, the chief requirement is a varying amount of manual dexterity, and tests of such dexterity would, in their case, probably be sufficient. For those engineering branches which require skilled apprentices, however, the qualities to be sought are a combination of intelligence, mechanical aptitude, ability, and dexterity. With that combination in mind, the report uses throughout the term 'apprentice ability'. Tests were therefore used which would depend inter alia on mental processes, but would be definitely associated with mechanical ability.

This primary difficulty of deciding the type of qualities to be sought in connexion with so wide an industry as engineering is, of course, well known to engineers. We stress it here not merely to show its complications, but also as an indication of the care with which the investigators dealt with their problem-a care which will go far towards building up methods and results upen which confidence may fairly be placed.

The groups of individuals selected included third and fourth year apprentices (average age nineteen years) attending part-time courses at the Birmingham Central Technical College; entrants and leavers (fourteen to sixteen years of age) of a school with an engineering bias (Handsworth Junior Day Technical School); of a school with a commercial bias (Aston Commercial School); and of a general secondary school (Yardley Secondary School).

This selection of pupils from differing types of postprimary schools possesses a special interest in view of the present reorganisation of education, which 